Case Report

\title{
A Chinese Child Presented with Early T Cell Precursor Lymphoblastic Lymphoma
}

\author{
Xiangyang Pu, ${ }^{1,2}$ Shengyong Deng, ${ }^{1,2}$ Nange Yin, ${ }^{1,3}$ Lin Song, ${ }^{3,4,5}$ Xiangling He, \\ and Jianwen Xiao $\mathbb{D}^{1,7,8}$ \\ ${ }^{1}$ Ministry of Education Key Laboratory of Child Development and Disorders, Chongqing, China \\ ${ }^{2}$ Department of Pediatric Respiratory Medicine, Qianjiang Central Hospital of Chongqing, Chongqing, China \\ ${ }^{3}$ Department of Pharmacy, Children Hospital of Chongqing Medical University, Chongqing, China \\ ${ }^{4}$ National Clinical Research Center for Child Health and Disorders, Chongqing, China \\ ${ }^{5}$ China International Science and Technology Cooperation Base of Child Development and Critical Disorders, \\ Chongqing 400014, China \\ ${ }^{6}$ Department of Pediatric Hematology and Oncology, \\ Children's Medical Center of Hunan Provincial People's Hospital (The First-Affiliated Hospital of Hunan Normal University), \\ Changsha, China \\ ${ }^{7}$ Chongqing Key Laboratory of Pediatrics, Chongqing, China \\ ${ }^{8}$ Department of Hematology, Children Hospital of Chongqing Medical University, Chongqing, China
}

Correspondence should be addressed to Jianwen Xiao; tomahawk6502@sohu.com

Received 20 May 2021; Revised 27 July 2021; Accepted 9 August 2021; Published 28 September 2021

Academic Editor: Saveria Capria

Copyright $\odot 2021$ Xiangyang Pu et al. This is an open access article distributed under the Creative Commons Attribution License, which permits unrestricted use, distribution, and reproduction in any medium, provided the original work is properly cited.

T cell lymphoblastic lymphoma (T-LBL) is regarded as the leukemic phase of T cell acute lymphoblastic leukemia (T-ALL). The early T cell precursors ALL/LBL (ETP-LBL/ALL) are derived from thymic cells at the ETP differentiation stage and recognized as a high-risk subgroup of T-ALL/LBL. Most of these cases presented with ALL at the disease onset, but the ETP-LBL phase is uncommon. Here, we report a patient who presented with ETP-LBL at the disease onset. In this case, ALL developed even despite receiving chemotherapy, but the patient achieved a complete remission with intensive chemotherapy.

\section{Introduction}

T cell lymphoblastic lymphoma (T-LBL) and T cell acute lymphoblastic leukemia (T-ALL) are both hematological tumors that originate from an immature $\mathrm{T}$ cell lineage. T-ALL is regarded as the leukemic phase of T-LBL, and a lymphoblast count in bone marrow $(\mathrm{BM})<25 \%$ is defined as the cutoff value between T-LBL and T-ALL $[1,2]$. The prognosis of T-LBL/ALL remains historically poor. ALLbased chemotherapy and allogenic hematopoietic stem cell transplantation (allo-HSCT) demonstrated effective results, and event-free survival (EFS) in children and adolescents exceeds $80-90 \%$ in developed countries [2, 3]. However, the survival of relapsed and/or refractory cases remains poor [2-4]. The early T cell precursors ALL/LBL (ETP-LBL/ALL) are derived from thymic cells at the ETP differentiation stage and are recognized as a high-risk subgroup of T-ALL/LBL [5]. ETP-LBL/ALL occurs in $10-12 \%$ or $20-25 \%$ of pediatric or adult T-LBL/ALL populations [5-7]. Most of these cases present with ALL, and the LBL phase is uncommon [6-8]. Here, we report a patient who presented with ETP-LBL at disease onset. ALL developed eventhough he subsequently received chemotherapy, and he achieved a complete remission (CR) with intensive chemotherapy.

\section{Case Presentation}

A 16-year-3-month-old boy was admitted with an irritating dry cough and cervical lymphadenopathy on 28 January 2020. During the physical examination, bilateral cervical 
lymph node masses were palpable, and signs of pleural effusion were also noted. Laboratory tests showed normal complete blood cell counts (white blood cells $6.22 \times 10^{9} / \mathrm{L}$, platelets $295 \times 10^{9} / \mathrm{L}$, and hemoglobin $161 \mathrm{~g} / \mathrm{L}$ ) and normal lactate dehydrogenase levels $(233 \mathrm{U} / \mathrm{L})$. A computed tomography (CT) scan revealed pericardial and right pleural effusion and lymphadenopathy at the cervical area bilaterally and within the mediastinum (Figure 1(a)). A core needle biopsy and subsequent immunohistochemical (IHC) staining of the mediastinum mass was performed for CD3+, CD20-, PAX5-, CD7+, CD34-, Ki-67+(90\%), MPO-, and CD99+(Figure 2). A sample of the pleural effusion was analyzed which showed that the nucleated cell counts were $31 \times 10^{9} / \mathrm{L}$, the lymphoblasts were $92 \%$, and there were $55.4 \%$ T cell lymphoblasts as detected by flow cytometry (FCM). Positive results for $\mathrm{CD} 7, \mathrm{CD} 13$, and $\mathrm{cCD} 3$ and negative results for CD1a, CD4, CD8, CD19, CD20, CD22, CD34, and MPO were confirmed (Figure 3). A diagnosis of ETP-ALL/ LBL was considered, and additional IHC samples of the mediastinum mass sample were stained via IHC and were tested. The results showed CD45pro-,CD5-, and CD79a were weak+, CD21 was positive, MUM-1 was negative, CD10 was positive, CK was negative, BCL6 was negative, c-MYC was $(40 \%+)$, CyclinD1 was negative, ALK was negative, CD30 was negative, and P53 was negative (Figure 2).

A chromosome karyotype of the pleural effusion sample was obtained using the International System of Human Cytogenetic Nomenclature 2009 (ISCN-2009) [2]: 43-50, X, $Y$, add(7) (p13), 9,?i(9) (q10), 14, 16, +22,?del(22) (q13), inc [CP8] (Figure 3). BM samples were acquired at the bilateral posterior superior iliac area, and blast cells were not detected by a BM smear, biopsy, or via FCM.

Samples were taken from the mediastinum mass, and whole exome sequencing (WES) and RNA sequencing (RNAseq) were performed. FBXW7 and GATA3 somatic mutations were detectable by WES (Table 1); transcripts of FBXW7, GATA3, JAK1, and NCOR1 were confirmed by RNAseq (Table 2).

A diagnosis of ETP-LBL was given based on previous literature reports [5-8], and the patient was classified as stage III by the revised International Pediatric NonHodgkin's Lymphoma Staging System [9]. He was treated with a modified BFM-LBL-95 protocol and was placed in an intermediate-risk (IR) group at the initial diagnosis [10]; the detail of the disease evaluation, risk group classification, treatment courses, dosages, and intrathecal injections had been listed at Supplementary Tables S1-S5, respectively; his treatment response was evaluated by BM, $\mathrm{CT}$, or positron emission tomography/computed tomography (PET/CT) imaging at different time points (TP) as the protocol required. On day 15 and day 33 (TP1 or TP2) during the course of the induction of remission I, the BM smear showed $29.5 \%$ and $80 \%$ blast cells, FCM had 29\% and 55.4\% ETP lymphoblasts (Figures 4(a) and 4(b)), and a chest $\mathrm{CT}$ scan revealed an unchanged mediastinum mass (Figure 1(b)).
He was considered to have progressive disease (PD) and refractory disease at TP2 $[5,6]$, and two standard courses A of the HyperCVAD protocol [11] were administered. After the 1 st course, lymphoblasts were no longer detected in the $\mathrm{BM}$ during the evaluation of the BM smear (Figure 4(c)); the CT scan showed a partial remission (PR) $[5,6]$, which was subsequently monitored by CT scans (Figure 1(c)). He achieved CR after the 2 nd course A, which was monitored by using BM smears (Figure 4(d)) and PET-CT scans (Figure 1(d)). The minimal residual disease (MRD) level was also detected by FCM [12], and a negative result was obtained $\left(<10^{-4}\right)$. Allo-HSCT was declined by the family, and he was treated with the CCLG-ALL-2008 protocol for the high-risk (HR) group [12]. Up until June 2021, he was alive without any evidence of a relapse.

\section{Discussion}

ETP-ALL/LBL is a recently described subgroup of T-ALL/LBL according to the World Health Organization criteria from 2016 (WHO 2016) [4]; usually, these cases are aggressive and present with ETP-ALL, whereas pediatric ETP-LBL is uncommon (20-30\% in adult T-ALL/LBL, pediatric data are absent). In the presented case, we reported a child who suffered from ETPLBL without BM infiltration at the onset.

ETP-ALL/LBL patients are distinguished from nonETPs by FCM and genomic signatures. ETP-ALL/LBL is immunophenotypically defined by weak or absent expression of T cell markers (CD1a, CD5, and CD8) and positive expression of at least one hematopoietic stem cell (HSC) and/or myeloid markers (CD13, CD33, CD34, CD117, and HLA-DR). The diagnosis of T-LBL was based on the pathological results and IHC staining, but not all the T cell, HSC, and myeloid markers were evaluated. Fortunately, the patient was diagnosed with ETP-ALL/LBL by FCM detected in a pleural effusion sample. FCM is an important technique to subclassify T-ALL/LBL, and all these T-ALL/LBL samples should be evaluated if possible.

Genomic signatures of ETP-ALL/LBL patients also exhibit unique genotypes compared with those of nonETPs patients $[7,8]$. Recurrent mutations were not only identified in the genes involved in T-lymphoid development or oncogenesis because myeloid markers were also detectable. Activating mutations encoding cytokine receptors and mediating the RAS signal transduction system (NRAS, KRAS, FLT3, and JAK1), inactivating mutations encoding transcription factors (GATA3, ETV6, and RUNX1) and histone repair (EZH2 and EP300) during hematopoietic stem cell development are common in ETPALL/LBL patients $[5,6,13]$. WES and RNAseq of LN samples have been evaluated, common gene mutations and transcripts (FBXW7, NOTCH1, and JAK1) in T-ALL/LBL have been identified, and unique genomic signatures of ETP-ALL/LBL have also been identified. This suggests that gene analysis can assist in the diagnosis of ETPs without using FCM detection. 

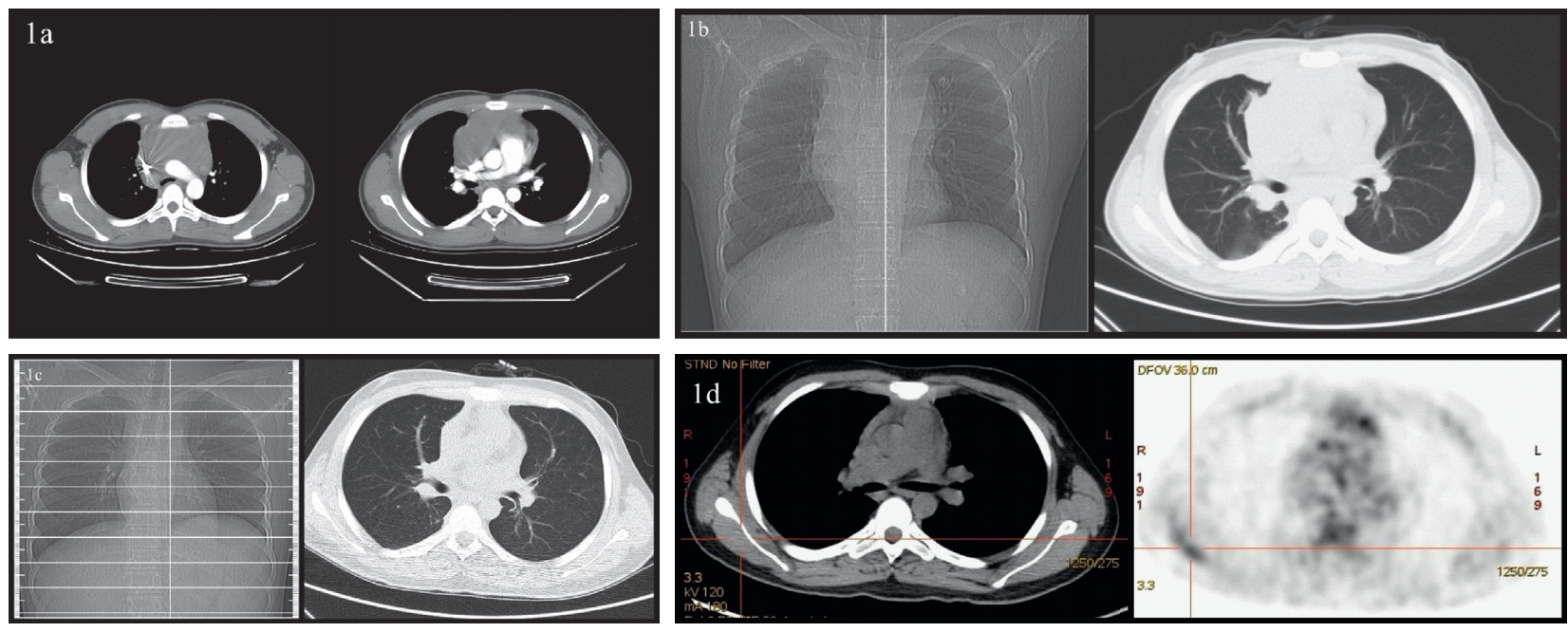

Figure 1: Results of chest CT or PET-CT scan.

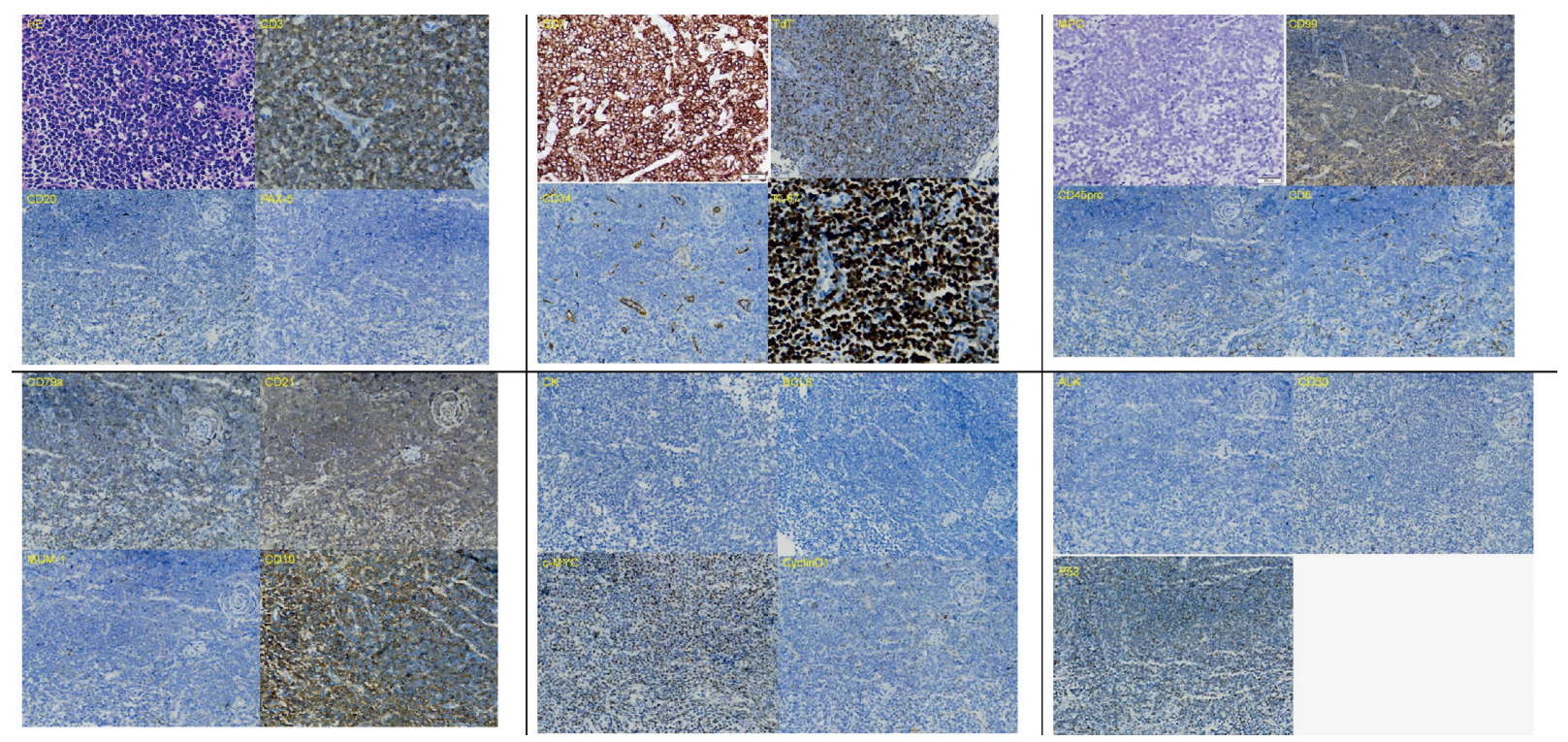

FIGURE 2: Results of mediastinum mass sample.

The best treatment and the prognosis for ETPs patients is unclear. Treatment responses and outcomes for ETPALL/LBL patients treated with chemotherapy were poorer than those for non-ETP-ALL/LBL adult patients, and it was reported that these cases benefit from allo-HSCT [4-6]. Although early reports of pediatric ETP-ALL showed a poor prognosis, recent clinical trials of COGAALL0434 and UKALL2003 showed that the prognosis of ETP-ALL was similar to that of other T-ALLs $[14,15]$. However, ETP-LBL was uncommon, but the treatment experience was limited. The patient in this case report was treated with a modified BFM-LBL-95 protocol, the ALL-based classic protocol for T-LBL patients, but he was considered to have refractory disease and infiltration into the BM. It was reported that adults benefited from the hyperCVAD protocol $[5,6]$, and in our patient, two courses A were administered, but course B was omitted due to financial constraints. The patient obtained CR, and chemotherapy was continued.

In conclusion, we reported a case of ETP-LBL that took an aggressive clinical course. Our case revealed that clinicians should pay attention to the possibility of ETP-LBL in common T-LBL cases, and FCM and genomic signatures are important to distinguish ETP-LBL. Further studies are needed to research the most effective treatment for ETPALL/LBL patients. 

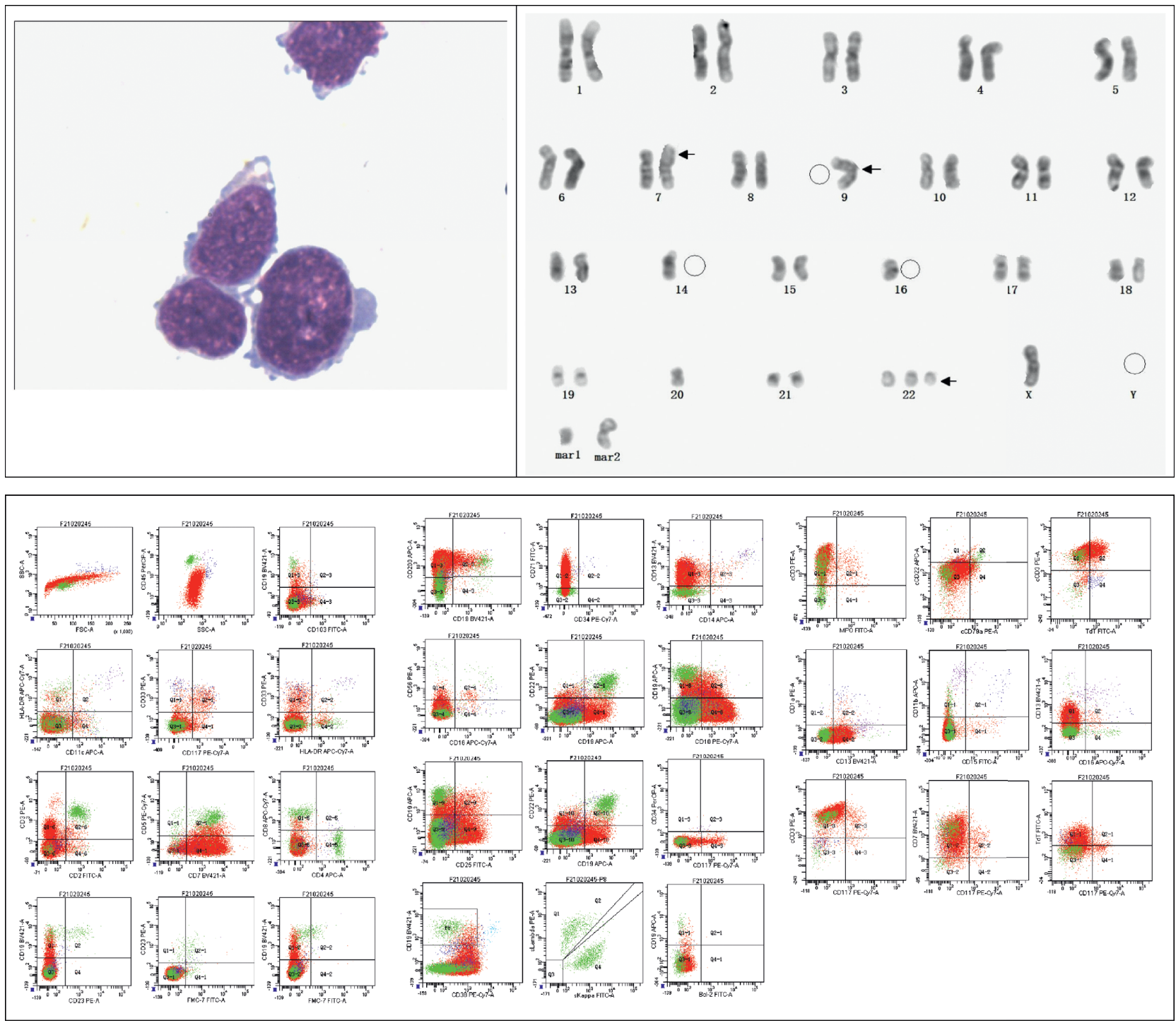

Figure 3: Results of pleural effusion sample.

TABLE 1: Results of whole exome sequencing.

\begin{tabular}{lccc}
\hline Genes & Chromosome coordinate & Mutation site & Mutation frequency (\%) \\
\hline FBXW7 & chr4: & c.1436G $>$ A & 38.3 \\
(NM_033632) & 153247366 & (p.Arg479Gln) & c.827G > A \\
GATA3 & chr10: & (p.Arg276Gln) & 38.8 \\
(NM_0010022) & 8106004 & c.811_812dup & \\
GATA3 & chr10: & (p.Thr272ArgfsTer24) & 26.7 \\
(NM_0010022) & 8105986 & \\
\hline
\end{tabular}

TABLE 2: Results of RNA sequencing.

Gene mutations

FBXW7 R479Q

GATA3 R276Q

GATA3 T270fs

JAK1 L783F

NCOR1 Y1435*

NOTCH1 V1721E
Mutation type

Missense mutation

Missense mutation

Frameshift mutation

Missense mutation

Truncating mutation

Missense mutation
Mutation frequency (\%)

51.9

68.8

24.4

58.5

34.6

34.1 


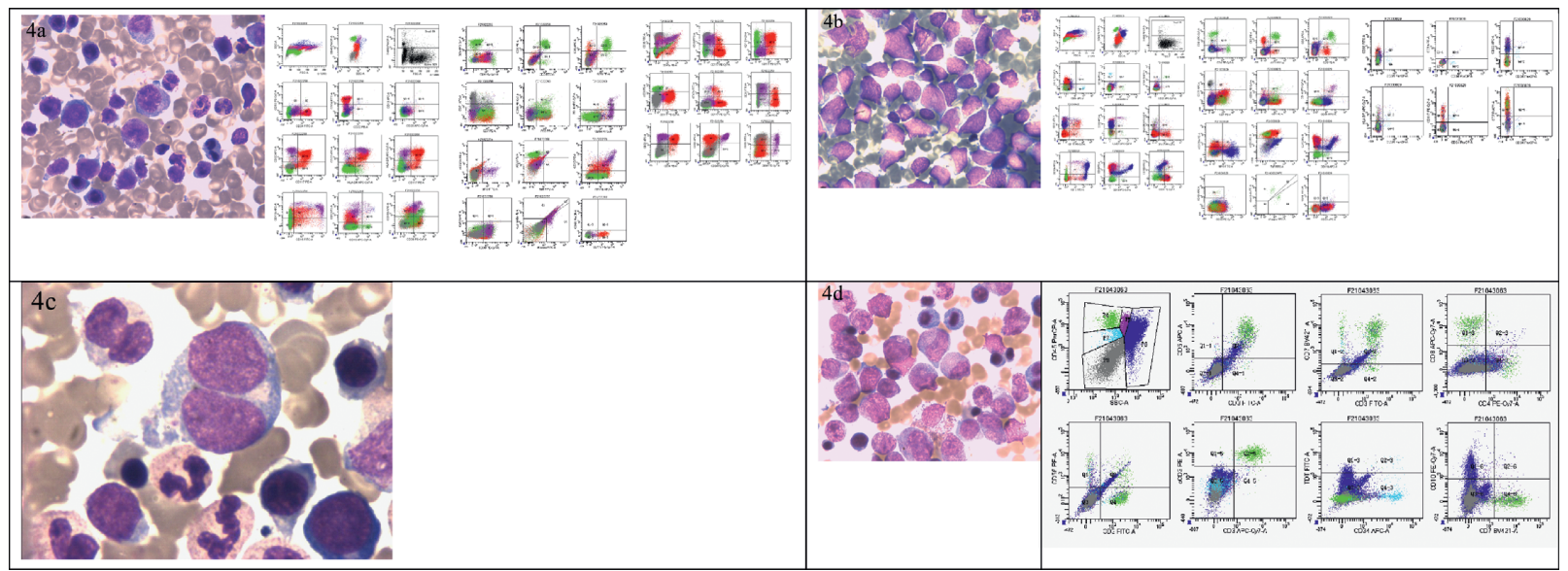

FIgURE 4: Results of bone marrow samples.

\section{Data Availability}

The data used to support the findings of this study are included within the article.

\section{Conflicts of Interest}

The authors declare that they have no conflicts of interest.

\section{Authors' Contributions}

JX, XP, and SD prepared the original draft. LS and JX helped with the design of the project. JX treated the patient and supervised the manuscript. All authors reviewed the final version.

\section{Acknowledgments}

The authors thank the patient and his family who participated in this study. This research was supported by the National Natural Science Foundation of China (Project no. 81900162) and the Chongqing Science and Technology Commission of PR China (Project no. cstc2018jsyjjsyjX0015).

\section{Supplementary Materials}

Table S1: disease evaluation. Table S2: risk group. Table S3: schedule of the protocol. Table S4: modified NHL-LBL-95 protocol. Table S5: schedule of intrathecal injections. (Supplementary Materials)

\section{References}

[1] B. Burkhardt and M. L. Hermiston, "Lymphoblastic lymphoma in children and adolescents: review of current challenges and future opportunities," British Journal of Haematology, vol. 185, no. 6, pp. 1158-1170, 2019.

[2] B. Burkhardt, S. Mueller, T. Khanam, and S. L. Perkins, "Current status and future directions of t-lymphoblastic lymphoma in children and adolescents," British Journal of Haematology, vol. 173, no. 4, pp. 545-559, 2016.
[3] M. J. You, L. J. Medeiros, and E. D. Hsi, "T-lymphoblastic leukemia/lymphoma," American Journal of Clinical Pathology, vol. 144, no. 3, pp. 411-422, 2015.

[4] W. Guan, Y. Jing, L. Dou, M. Wang, Y. Xiao, and L. Yu, "Chidamide in combination with chemotherapy in refractory and relapsed T lymphoblastic lymphoma/leukemia," Leukemia and Lymphoma, vol. 61, no. 4, pp. 855-861, 2020.

[5] N. Jain, A. V. Lamb, S. O’Brien et al., "Early T-cell precursor acute lymphoblastic leukemia/lymphoma (ETP-ALL/LBL) in adolescents and adults: a high-risk subtype," Blood, vol. 127, no. 15, pp. 1863-1869, 2016.

[6] J. Bond, C. Graux, L. Lhermitte et al., "Early response-based therapy stratification improves survival in adult early thymic precursor acute lymphoblastic leukemia: a group for research on adult acute lymphoblastic leukemia study," Journal of Clinical Oncology, vol. 35, no. 23, pp. 2683-2691, 2017.

[7] M. Ma, X. Wang, J. Tang et al., "Early T-cell precursor leukemia: a subtype of high risk childhood acute lymphoblastic leukemia," Frontiers of Medicine, vol. 6, no. 4, pp. 416-420, 2012.

[8] Y. Jiang, A. Maiti, and Z. Kanaan, "A young man with a mass: non-early precursor t-cell lymphoblastic lymphoma," The American Journal of Medicine, vol. 132, no. 1, pp. 58-60, 2019.

[9] A. Rosolen, S. L. Perkins, C. R. Pinkerton et al., "Revised international pediatric non-hodgkin lymphoma staging system," Journal of Clinical Oncology, vol. 33, no. 18, pp. 2112-2118, 2015.

[10] C. Y. Zheng, J. W. Xiao, J. Yu, and Y. G. Chen, "Prognosis and side effects of T-NHL-2009 protocol on treatment of lymphoblastic lymphoma in Children," Clinics in Laboratory Medicine, vol. 14, no. 13, pp. 1883-1887, 2017.

[11] C. R. Rausch, E. J. Jabbour, H. M. Kantarjian, and T. M. Kadia, "Optimizing the use of the hyperCVAD regimen: clinical vignettes and practical management," Cancer, vol. 126, no. 6, pp. 1152-1160, 2020.

[12] L. Cui, Z.-G. Li, Y.-H. Chai et al., "Outcome of children with newly diagnosed acute lymphoblastic leukemia treated with CCLG-ALL 2008: the first nation-wide prospective multicenter study in China," American Journal of Hematology, vol. 93, no. 7, pp. 913-920, 2018.

[13] O. C. Puglianini and N. Papadantonakis, "Early precursor T-cell acute lymphoblastic leukemia: current paradigms and evolving concepts," Therapeutic Advances in Hematology, vol. 11, Article ID 2040620720929475, 2020.

[14] S. S. Winter, K. P. Dunsmore, M. Devidas et al., "Improved survival for children and young adults with t-lineage acute 
lymphoblastic leukemia: results from the children's oncology group AALL0434 methotrexate randomization," Journal of Clinical Oncology: Official Journal of the American Society of Clinical Oncology, vol. 36, no. 29, pp. 2926-2934, 2018.

[15] K. Patrick, R. Wade, N. Goulden et al., "Outcome for children and young people with early t-cell precursor acute lymphoblastic leukaemia treated on a contemporary protocol, ukall 2003," British Journal of Haematology, vol. 166, pp. 421-4, 2014. 